\title{
INDÚSTRIA 4.0 E OS DESAFIOS DA EDUCAÇÃO
}

Fernando Covolan Rosito-e-mail: fernando.rosito@farroupilha.ifrs.edu.br Instituto Federal de Educação, Ciência e Tecnologia do Rio Grande do Sul (IFRS) Av. São Vicente, 785 - Bairro Cinquentenário CEP: 95174-274 - Cidade: Farroupilha - Estado: RS

Eliana Maria do Sacramento Soares-e-mail: emsoares@ucs.br Universidade de Caxias do Sul (UCS)

Rua Francisco Getúlio Vargas, 1130

CEP: 95070-560 - Cidade: Caxias do Sul-Estado: RS

Carine Geltrudes Webber-e-mail: CGWebber@ucs.br

Universidade de Caxias do Sul (UCS)

Rua Francisco Getúlio Vargas, 1130

CEP: 95070-560 - Cidade: Caxias do Sul-Estado: RS

Resumo: A indústria 4.0, identificada como a última revolução industrial, vem modificando o ambiente fabril e social em diversos aspectos, desde a produção até a aplicação das tecnologias emergentes no cotidiano das pessoas. A manufatura avançada, fazendo uso de máquinas inteligentes que compartilham informações e tomam decisões sem intervenção humana, determinam as novas habilidades e competências necessárias para alguns profissionais. Na área da Engenharia, as inovações da indústria 4.0 se fazem presente, pois envolvem conhecimentos técnicos e científicos, exigindo uma atualização constante das operações industriais e de mercado. Neste contexto, o presente texto apresenta uma pesquisa sobre desafios, habilidades e competências atreladas à formação de engenheiros para atuarem na indústria 4.0, enfatizando a necessidade de renovação educacional envolvendo tecnologias emergentes. Nesta direção, o trabalho tem por objetivo provocar uma reflexão sobre a inserção de alguns conceitos e tecnologias, utilizadas na indústria 4.0, nos currículos. Como resultado, identifica-se o quão importante é haver o alinhamento de ideias e de ações entre governo, empresas, instituições de educação e sociedade para que ocorra uma reformulação do ensino por meio da qualificação continuada de professores e da estruturação de laboratórios das escolas.

Palavras-chave: Educação. Indústria 4.0. Engenharia.

\section{INTRODUÇÃO}

Atualmente, o cenário industrial está passando por mudanças tecnológicas significativas, projetando novas tendências em diversos segmentos, inclusive no âmbito da Engenharia. Este fato influencia no desenvolvimento acadêmico das instituições de ensino, que necessitam aprimorar suas práticas pedagógicas a fim de aperfeiçoar as habilidades dos estudantes. Isto se faz necessário à medida que se observa carências na formação dos engenheiros para atenderem as demandas do mercado de trabalho de uma indústria em fase de reestruturação mundial. 
As transformações sociais, decorrentes da expansão das tecnologias, implicam em atualizações de competências do indivíduo. Ou seja, a pessoa, hoje, utiliza muitos recursos eletrônicos para a sua integração comunitária, como, por exemplo, o uso de smartphone para enviar e receber mensagens, substituindo as cartas escritas à mão e expedidas pelo correio. Também está sendo comum empresas de serviços comercializarem produtos e resolverem problemas por meio de sistemas automatizados via internet. Muitas dessas atividades utilizam tecnologias avançadas, como os serviços que fazem uso de computadores baseados em inteligência artificial (IA) para interagir com o cliente via chatbots. Chatbots são as caixas de bate-papo na página inicial de sites de comércio eletrônico e aplicativos móveis programados para se comunicar com os clientes de maneira personalizada (OLIVEIRA, 2020). Devido a esses cenários, é possível perceber que a sociedade está cada vez mais envolvida e dependente de recursos tecnológicos.

Já no âmbito de produção industrial, o processo de globalização, impulsionado pela evolução tecnológica da indústria 4.0, tem alterado os processos de inserção e manutenção das empresas no mercado. Tal fator tem exigindo uma constante transformação das indústrias em seus parques fabris, gerando inovações tecnológicas. Cita-se, por exemplo, o uso de robôs autônomos no setor de estoques, que fazerem a separação de produtos que serão enviados aos clientes, com o mínimo de intervenção humana. Contudo, para se manterem fortes perante os concorrentes, as empresas buscam progressos nos processos e produtos, melhorias de serviço ao cliente, redução de custos, diminuição do tempo de lançamento de produtos, geração de novos modelos de negócios, entre outros fatores (BRASIL, 2017). Essa necessidade de inovação e melhorias contínuas com o uso de tecnologias impacta na exigência de habilidades e competências dos seus colaboradores, entre eles, os engenheiros.

Para que um profissional possa se inserir de forma crítica, reflexiva e produtiva na sociedade, ela necessita acompanhar o desenvolvimento tecnológico, pois, como visto, atualmente se utiliza muitos recursos inovadores tanto nas indústrias como no cotidiano. $\mathrm{O}$ indivíduo, querendo ou não, está imerso em um universo tecnológico. Desse modo, o aprimoramento e a atualização constante de habilidades e competências são importantes para o profissional continuar produzindo conteúdo intelectual. Para isso, a educação tem um papel fundamental, pois com ela é possível realizar esta adequação de conhecimentos, promovendo uma formação cidadã-tecnológica, principalmente na área da Engenharia, que está fortemente envolvida com inovações científicas de produção industrial. Portanto, é necessário observar a evolução tecnológica que transforma a sociedade, alterando o perfil dos trabalhadores e os costumes das pessoas em relação ao uso de tecnologias. Esse acompanhamento é essencial para desenvolver estratégias de ensino-aprendizagem voltadas para formação de engenheiros (e de outras áreas) com o intuito de prolongar a vida útil das organizações e atender demandas futuras.

Constantes avaliações curriculares já foram realizadas ao longo da vida dos cursos de Engenharia. Todas elas buscaram acompanhar as mudanças na sociedade e nos setores produtivos. Nos últimos anos nota-se que as transformações têm ocorrido com maior rapidez e abrangência, forçando as indústrias a inovarem, sob pena de tornarem-se obsoletas (PEARSON, 2011). Tais carências repercutem nos cursos de Engenharia e, sendo assim, este trabalho tem por objetivo provocar uma reflexão sobre a inserção de alguns conceitos e tecnologias, utilizadas na indústria 4.0, nos currículos. Neste sentido, deve-se repensar, discutir e refinar propostas que viabilizem a formação dos engenheiros que irão atuar nos cenários da indústria 4.0. Para isso, este texto propõe uma revisão da história e dos conceitos de Indústria 4.0, em seguida aponta desafios, habilidades e competências que precisam ser abordados na formação de engenheiros e, a partir deste ponto, sinaliza caminhos que podem ser explorados em busca do objetivo proposto. 


\section{REVOLUÇÃO INDUSTRIAL E TECNOLOGIAS EMERGENTES}

As revoluções industriais dizem respeito, basicamente, as mudanças significativas no modo de fabricação dos produtos. Nesse sentido, houve quatro revoluções industriais, sendo que a primeira, conhecida como indústria 1.0, ocorreu devido à mudança de fabricação artesanal pela mecanização da produção pelas máquinas a vapor na segunda metade do século XVIII (HEINDL et al., 2016). A segunda revolução industrial (indústria 2.0) originou-se pela utilização da energia elétrica nos processos produtivos e pela massificação da produção, ocorrida após a metade do século XIX (MDIC; ABDI, 2018; BAYGIN et al., 2016). Já a terceira revolução diz respeito à automatização de atividades mecânicas e repetitivas através de máquinas com suporte eletrônico. Nesse período a eletrônica, os computadores, a internet e a tecnologia da informação (TI) foram recursos utilizados para o processo de produção de produtos nas fábricas (BAYGIN et al., 2016; HEINDL et al., 2016). Muitos países em desenvolvimento ainda utilizam este cenário como meio tecnológico de produção. Outros, por sua vez, estão utilizando as chamadas fábricas inteligentes, provenientes do contexto da $4^{\mathrm{a}}$ revolução industrial, também conhecida como indústria 4.0, manufatura avançada, entre outros nomes (BAYGIN et al., 2016). Essa última revolução industrial está relacionada a uma maior conectividade das máquinas e processos de produção em tempo real, viabilizando o gerenciamento automático de um grande fluxo de informações entre diversos segmentos. Como consequência, permite a personalização da produção em massa. Nesse contexto, máquinas coletam dados, compartilham informações de produção, distribuem e executam tarefas com pouca ou nenhuma intervenção humana (FORSCHUNGSUNION; ACATECH, 2013; HEINDL et al., 2016). Esse tipo de inovação industrial compreende várias tecnologias que são importantes para as pessoas, visto que muitas delas são empregadas no contexto industrial e social, estando presentes em dispositivos eletrônicos que usamos frequentemente.

$\mathrm{Na}$ indústria 4.0, o surgimento de sistemas inteligentes e conectados no âmbito físico e virtual (digital), denominados sistemas ciber-físicos, representam o centro da fábrica inteligente do futuro. Nesses processos, existe a preocupação não somente por uma produtividade mais elevada, mas também por uma eficiência energética (HEINDL et al., 2016). Para Forschungsunion e Acatech (2013) os sistemas ciber-físicos compreendem máquinas inteligentes e sistemas de produção capazes de compartilhar informações, desencadeando ações e controles independentes.

Tais processos de fabricação inteligentes são complexos, envolvendo muitas outras tecnologias da indústria 4.0, como, por exemplo, as técnicas de inteligência artificial (IA). Considerando os avanços nos métodos e procedimentos da IA é possível que as máquinas simulem a capacidade humana de raciocinar, aprender continuamente e resolver problemas de forma autônoma (MDIC; ABDI, 2018). Graças ao avanço dessa tecnologia, especialistas acreditam que a inteligência das máquinas se equiparará à dos humanos até 2050 (ALCOFORADO, 2019).

O relatório intitulado "Indústria 4.0: O Futuro da Produtividade e Crescimento nas Indústrias de Manufatura" (Industry 4.0: The Future of Productivity and Growth in Manufacturing Industries) do Boston Consulting Group $(B C G)^{1}$ prevê nove pilares tecnológicos utilizados na indústria 4.0 que devem emergir na fabricação nas próximas décadas. Estas tecnologias são: robôs autônomos, simulação, integração de sistemas, internet das coisas,

\footnotetext{
${ }^{1}$ Boston Consulting Group (BCG) é especialista em estratégia de negócios, o qual associa líderes empresariais e sociais para enfrentar seus desafios mais importantes e capturar suas maiores oportunidades (RÜßMANN et al., 2015).
} 
cibersegurança, computação em nuvem, manufatura aditiva, realidade aumentada e análise de big data. Esses pilhares são vistos como determinantes para a produtividade e crescimento das indústrias sobre esta nova configuração tecnológica (RÜßMANN et al., 2015). Contudo, todos envolvem profissionais preparados para atuarem com ferramentas e processos tecnológicos altamente sofisticados e complexos.

Além dessas tecnologias citadas, existem outras que estão sendo desenvolvidas graças a indústria 4.0, tais como a biologia sintética, a internet of services $(I o S)$, veículos autônomos, nanomateriais e nanosensores (MDIC; ABDI, 2018; TESSARINI JUNIOR; SALTORATO, 2018). O documento "Plano de ação de ciência, tecnologia e inovação para tecnologias convergentes e habilitadoras" do Ministério da Ciência, Tecnologia, Inovações e Comunicações (MCTIC), prevê os cenários possíveis da aplicação das tecnologias para manufatura avançada, sendo estes: segurança, robótica e automação, conectividade e integração, big data/cloud \& analytics, agricultura inteligente, internet das coisas, manufatura inteligente, mineração inteligente, sensores/bateria, manufatura aditiva e, realidade virtual e aumentada. $\mathrm{O}$ documento enfatiza que algumas áreas são mais factíveis e outras um pouco distantes para o Brasil, mas sem dúvida todas estarão presentes com o tempo (BRASIL, 2020).

Assim, pode-se perceber que há muitos recursos tecnológicos sendo mais explorados e estudados pela eminência da $4^{\text {a }}$ revolução industrial. Em um cenário industrial em que as máquinas compartilham informações e tomam decisões sobre problemas de produção com o mínimo de intervenção humana, é possível vislumbrar a repercussão da manufatura avançada no mundo do trabalho. Sendo assim, é considerável a preocupação com a formação dos futuros profissionais, dada a complexidade das tecnologias empregadas pela indústria 4.0. A conexão entre as pessoas e as tecnologias deve ocorrer durante as suas vidas acadêmicas com a inclusão desses assuntos nas grades curriculares. Por esta razão, conhecer o novo perfil dos engenheiros e de outros profissionais que trabalham com essas tecnologias emergentes é importante, uma vez que este pode ser o ponto de partida para redimensionar a estrutura dos cursos para profissionais dessa área.

\section{DESAFIOS, HABILIDADES E COMPETÊNCIAS DE ENGENHEIROS E OUTROS PROFISSIONAIS PARA O CENÁRIO TECNOLÓGICO EMERGENTE}

Segundo Instituto SENAI de Inovação em Sistemas Virtuais de Produção (2019) o Brasil está passando por um processo de transformações no ambiente industrial devido ao avanço tecnológico e estima-se que 15,7 milhões de postos de trabalho serão afetados até 2030 (INSTITUTO SENAI DE INOVAÇÃO EM SISTEMAS VIRTUAIS DE PRODUÇÃO, 2019). As tecnologias da indústria 4.0 influenciam na interação homem-máquina, mudando também as exigências enfrentadas pelos empregados. Por esse motivo, o cotidiano profissional está caracterizado por tarefas menos braçais e mais direcionadas para coordenação e monitoramento de sistemas inteligentes (HEINDL et al., 2016). É importante a reflexão, pois algumas das consequências da evolução industrial são o declínio de empregos referente às tarefas simples e repetitivas, por serem mais suscetíveis à automação e; o surgimento de novos cargos em níveis gerenciais ou em áreas que exigem maior qualificação (ALCOFORADO, 2019; TESSARINI JUNIOR; SALTORATO, 2018). Deste modo, diversos profissionais, inclusive da engenharia, estarão envolvidos nesse contexto.

O "Mapa do Trabalho Industrial 2019-2023", elaborado pelo Serviço Nacional de Aprendizagem Industrial (SENAI) a partir de cenários sobre o comportamento da economia brasileira e dos seus setores, prevê que as mudanças tecnológicas para a fabricação de produtos demandará gradativamente mais especialistas na área de implementação de processos 
robotizados (BOAVENTURA, 2019). Nessa evolução, a tendência é que as empresas invistam em máquinas e programas mais avançados, sendo promissoras as projeções para os engenheiros, principalmente de Controle e Automação. Como a evolução industrial vislumbra a crescente eficiência e a inserção tecnológica nos processos de produção, pode-se dizer que esse profissional será bastante requisitado nos próximos anos (SERGS, 2018).

Conforme o mesmo mapa do trabalho industrial, as maiores taxas de crescimento de empregos serão de ocupações que têm como base a tecnologia, por exemplo: pesquisadores de Engenharia e Tecnologia (aumento de 17,9\%); engenheiros de Controle e Automação, engenheiros Mecatrônicos e afins (14,2\%), entre outros (BOAVENTURA, 2019). Porém, a Sociedade de Engenharia do Rio Grande do Sul (SERGS) revela um fato intrigante na engenharia, que é o desvio da área de formação devido ao pouco incentivo empresarial para com os engenheiros. Assim, esses acabam atuando em outros setores. A mesma fonte reforça que para reter talentos é preciso ofertar salários atrativos e capacitação profissional. Ao encontro dessa informação, o Instituto de Pesquisa Econômica Aplicada (IPEA) mostrou que menos da metade (41\%) dos engenheiros exercem suas ocupações típicas (SERGS, 2018). Logo, esses dados apontam que o engenheiro possui versatilidade, mas que há um desafio das indústrias em cativar este profissional para desempenhar atividades de sua formação.

As empresas procuram cada vez mais profissionais com competências diversificadas, ou seja, que não tenham apenas conhecimentos técnicos, mas também habilidades em se comunicar e trabalhar em equipe. Desta maneira, os engenheiros necessitam incluir em seus currículos as atribuições referentes a relacionamentos interpessoais. Nos últimos anos o mercado espera que os profissionais já integrem as equipes rapidamente com essas habilidades consolidadas, investindo, assim, em treinamentos e capacitações profissionais mais na área técnica (SERGS, 2018). Segundo a pesquisa de Silva, Kovaleski e Pagani (2019), as competências bases para pessoas na indústria 4.0 são: comunicação, criatividade, inovação, processos decisórios, habilidades analíticas, liderança e conhecimento técnico. Já Tessarini Junior e Saltorato (2018) identificaram, através de suas pesquisas, três categorias relacionando as competências requeridas pelas fábricas inteligentes, sendo estas: competências funcionais, entendidas como aquelas necessárias para o desempenho técnico e profissional das tarefas; competências comportamentais, mais intrínsecas e relacionadas às atitudes do indivíduo e; competências sociais, relacionadas com a capacidade de interagir e trabalhar com outras pessoas.

O estudo de Alcoforado (2019) indica quatro habilidades que são essenciais em um ambiente com tecnologias avançadas: a inteligência interpessoal, uma habilidade de se relacionar com outras pessoas, destacando-se a capacidade de criar empatia e liderança; a inteligência intrapessoal, que envolve a capacidade de se conhecer e relacionar consigo, destacando-se o autoconhecimento, autocontrole e domínio de emoções; a inteligência interartificial, que é a habilidade de compreender o impacto da tecnologia, isto é, conhecer as potencialidades das novas tecnologias, como robótica e inteligência artificial, e utilizá-las como ferramentas a nosso favor e; por fim, a inteligência criativa, que é, por enquanto, o principal diferencial entre a inteligência humana e a artificial, ou seja, a capacidade de criar algo novo utilizando as demais inteligências e aplicando-as de forma inovadora. Rajnai e Kocsis (2017) apontam características ocupacionais no processo de digitalização e informatização, como: a percepção e manipulação das tecnologias; a inteligência criativa (originalidade, belas artes) e; a inteligência social (percepção, habilidades de negociação, cooperação com outras pessoas).

Além de conhecer as habilidades e competências das pessoas sobre a indústria 4.0, também é importante saber como desenvolver esses atributos junto com o ser humano. Assim, conforme estudo de Silva, Kovaleski e Pagani (2019), a qualificação deve ocorrer por meio da capacitação de pessoas, treinamentos integrados em laboratórios técnicos providos de tecnologias 
adequadas (hardwares e softwares), entre outras ações. O estudo também revela a importância das competências de TI e de pensamento humano interdisciplinar como elementos curriculares básicos para os estudantes. Desta maneira, a qualificação para a manufatura avançada é um dos requisitos primordiais para o sucesso desta $4^{a}$ revolução industrial. $\mathrm{O}$ estudo enfatiza que a participação das pessoas continuará sendo necessária, uma vez que nem todas as tarefas poderão ser substituídas por sistemas ou máquinas com inteligência artificial. O que irá ocorrer é um redirecionamento de atividades, ou seja, os profissionais desenvolverão outras tarefas, como, por exemplo, a programação de robôs nas linhas de montagem, operações de sistemas, supervisões gerais e trabalhos com processos decisórios. Mesmo que muitas empresas brasileiras ainda vivenciam a $2^{\mathrm{a}}$ revolução industrial, conforme o documento "Plano de CT\&I para Manufatura Avançada no Brasil ProFuturo - Produção do Futuro" do MCTIC, é importante o país se preocupar e investir em uma educação voltada para as tecnologias emergentes (BRASIL, 2017).

Então, para que ocorra o desenvolvimento das competências e habilidades de uma pessoa de forma a promover o potencial humano nas organizações e suprir as necessidades da $4^{\mathrm{a}}$ revolução industrial, existem, segundo Tessarini Junior e Saltorato (2018), duas principais estratégias possíveis: aprendizagem contínua e à inovação constante no ambiente de trabalho e; a reformulação nos sistemas educacionais, conciliando os interesses públicos, privados e científicos (TESSARINI JUNIOR; SALTORATO, 2018). Assim, setores empresariais, governamentais, acadêmicos e a sociedade devem criar políticas direcionadas à formação profissional nos diversos níveis de aprendizagem, assim como de questões relativas ao trabalho humano nas indústrias (BRASIL, 2017; SILVA; KOVALESKI; PAGANI, 2019).

\section{A EDUCAÇÃO DIRECIONADA PARA AS TECNOLOGIAS EMERGENTES}

Segundo o "Mapa do Trabalho Industrial 2019-2023”, desenvolvido pelo SENAI, será necessário qualificar 10,5 milhões de trabalhadores em ocupações industriais para atender às demandas do mercado de trabalho (BOAVENTURA, 2019). Para entender a realidade de cada região, o SENAI também estruturou um mapa do trabalho industrial de cada um dos 26 estados e do Distrito Federal. Assim, conforme o estudo, o Rio Grande do Sul terá de qualificar mais de 800 mil trabalhadores em ocupações industriais nos níveis de formação superior, técnico e aperfeiçoamento entre 2019 e 2023 (FIERGS, 2019).

Devido à ascensão da tecnologia ao longo do tempo, o ensino que está sendo ofertado nos moldes atuais provavelmente será ineficaz daqui a alguns anos. Caso não ocorra uma renovação na educação, os sistemas de ensino estarão preparando estudantes e profissionais para um mundo (social e de trabalho) que deixará de existir (ALCOFORADO, 2019). Assim, algumas ações possíveis para a mudança de paradigma na educação são: estimular a formação e qualificação de professores para com soluções pedagógicas adequadas ao desenvolvimento de competência e habilidades em tecnologias emergentes (ALCOFORADO, 2019; BRASIL, 2017); incentivar à integração de professores e alunos em atividades empresariais de manufatura avançada (BRASIL, 2017); realizar o acompanhamento de universidades, colégios e outras instituições de educação em relação aos seus procedimentos de ensino e; incentivar as empresas pela formação continuada e treinamento dos funcionários (HEINDL et al., 2016).

Como exemplos de adaptação da educação, podem-se citar países como a Suíça e a Finlândia, que já começaram a considerar a nova realidade tecnológica e industrial, iniciando o processo de adequação de suas sociedades. Assim, começaram pela reformulação de seus sistemas educacionais, privilegiando: o desenvolvimento da habilidade de metacognição, ou seja, a capacidade do ser humano ter consciência de seus atos e pensamentos; o domínio de 
idiomas (em especial da língua inglesa, devido ao fato de que a maioria do conhecimento humano está registrado nesse idioma) e; um currículo baseado em Ciências, Tecnologia, Engenharia e Matemática, oriunda da sigla em inglês STEM (Science, Technology, Engineering, and Mathematics), associado ao método de "arte liberal" em que é possível formar uma mentalidade mais direcionada à criação de propriedade intelectual e a imaginação (para atuar criativamente na sociedade e gerar inovação) (ALCOFORADO, 2019).

Rajnai e Kocsis (2017) elencam ações necessárias para a mudança no sistema de treinamento e educação em relação à digitalização (processo valioso na indústria 4.0), sendo estas: fortalecer a área de STEM; proporcionar ao estudante conhecimentos e habilidades para mudanças contínuas; manter o sistema de educação atualizado, aderindo currículos interdisciplinares no ensino superior; introduzir novas formas de educação, incluindo a aprendizagem ao longo da vida, cursos abertos e online, cooperação da empresa e instituições de ensino.

A pesquisa de Alarcon et al. (2018) destaca três ambientes pedagógicos para trabalhar com alta tecnologia, possibilitando a integração da interdisciplinaridade, processos de ensinoaprendizagem e a automação das indústrias, sendo estas: utilização da internet das coisas; laboratórios remotos de robótica e; laboratórios de fabricação (experimentação e inovação), denominados Fab-Lab. Com essas ferramentas o aluno tem ao seu alcance espaços de experimentação e aprendizagem para o desenvolvimento de soluções automatizadas com o uso de peças de robótica, impressoras 3D, peças e componentes para usos diversos, entre outros dispositivos digitais que também são evidenciados no cenário da indústria 4.0.

É importante salientar que neste ano de 2020, para agravar a situação em todas as esferas sociais e econômicas, o mundo enfrenta o novo Corona vírus, causador de uma doença (COVID-19) que não tem vacina neste momento. Apesar de a pandemia ser um fator negativo para todos, ela demanda esforços e criatividade, principalmente por parte dos pesquisadores, para inovar e resolver problemas da sociedade. $\mathrm{O}$ uso de tecnologias utilizadas na indústria 4.0 pode ser benéfico durante a crise, ajudando no desenvolvimento de soluções em todas as áreas, como na área da Saúde. Assim, os robôs podem auxiliar na prevenção da doença, já que esses têm o potencial de desinfectar superfícies e a capacidade de fornecer medicamentos e alimentos aos pacientes. Também podem ser utilizados para realizar diagnósticos e triagem de pessoas contaminadas através da medição de temperatura e outros dos sinais vitais, auxiliando no controle em áreas de risco. Deste modo, o impacto do COVID-19 pode impulsionar mais pesquisas em robótica para abordar riscos de doenças infecciosas (YANG et al., 2020).

\section{CONSIDERAÇÕES FINAIS}

A partir das considerações apresentadas destacamos que a indústria 4.0 envolve diversas tecnologias e provoca mudanças no âmbito social e industrial. A revolução tecnológica impacta nos empregos, incluindo da engenharia, uma vez que são exigidas novas habilidades e competências das pessoas para o novo cenário industrial. Por esse motivo, a educação deve sofrer alterações para fortalecer e aproximar a sociedade às tecnologias emergentes, já que além de promover o pensamento crítico e autônomo, a escola pode capacitar o aluno a desenvolver soluções com o auxílio da alta tecnologia. Para a engenharia, os conceitos da indústria 4.0 são bastante relevantes, dado que a área tem grande aproximação com a indústria.

Esta renovação da educação, que aplica tecnologias desenvolvidas na $4^{\mathrm{a}}$ revolução industrial, proporciona à sociedade uma oportunidade de pessoas enfrentarem os desafios oriundos da manufatura avançada. Porém, é preciso observar as rápidas mudanças que acontecem no mundo em relação aos avanços tecnológicos, assim como acompanhar as 
habilidades e competências necessárias para o trabalho do século XXI. Desse modo, é fundamental a reflexão sobre a estrutura escolar oferecida aos alunos e a reavaliação curricular das instituições de ensino, devendo permitir a formação de estudantes para que saibam trabalhar em equipe, com capacidade técnica para executar projetos reais, com aptidão para decidir ações, entre outras habilidades. Para tal resultado, a base dos conteúdos sobre a manufatura avançada deve ser temática nos currículos dos diversos níveis de ensino, mostrando que o investimento deve ser sistêmico, desde o início escolar do indivíduo. Também são necessárias a formação e requalificação de professores com soluções pedagógicas adequadas voltadas para o desenvolvimento de competências e habilidades em manufatura avançada; além da infraestrutura apropriada para a implementação de laboratórios com alta tecnologia e conectividade.

O presente trabalho também revela que essa transformação na educação depende da união do governo, empresas, escolas e da própria sociedade. Através dessa integração é possível gerar ambientes colaborativos de ensino, em que empresas e escolas possam dividir um espaço tecnológico, permitindo a criação ou o enriquecimento do maquinário voltado para as novas tecnologias, assim como promover treinamentos e qualificação em conjunto. Engenheiros, professores e estudantes, com o auxílio de seus governantes, são primordiais para a reformulação da educação da Engenharia. Com momentos de reflexões e ações conjuntas será mais fácil desenvolver uma sociedade mais preparada para o futuro.

\section{Agradecimentos}

Gostaríamos de agradecer ao Instituto Federal de Educação, Ciência e Tecnologia do Rio Grande do Sul (IFRS) e à Universidade de Caxias do Sul (UCS) pelo apoio dado para o desenvolvimento da pesquisa realizada.

\section{REFERÊNCIAS}

ALARCON, D. F. et al. Os Desafios da Educação em Rede no Contexto da Indústria 4.0. In: SANTOS, C. C. (org.). Estudos interdisciplinares nas ciências exatas e da terra e engenharias 3. Ponta Grossa: Atena Editora, 2018. cap. 26, p. 279-293. Disponível em: https://www.atenaeditora.com.br/wp-content/uploads/2019/09/E-BOOK-Estudos-

Interdisciplinares-nas-Ciencias-Exatas-e-da-Terra-e-Engenharias-3-1-1-1.pdf. Acesso em: 19 nov. 2019

ALCOFORADO, F. O Futuro do Trabalho e da Educação no Mundo. Revista Científica de Investigación Educativa de La Unae RUNAE, p. 44 - 65, 2019. Disponível em: http://revistas.unae.edu.ec/index.php/runae/issue/view/16/42. Acesso em: 23 nov. 2019.

BAYGIN, M. et al. An Effect Analysis of Industry 4.0 to Higher Education. In: 15TH INTERNATIONAL CONFERENCE ON INFORMATION TECHNOLOGY BASED HIGHER EDUCATION AND TRAINING (ITHET), 2016, Istanbul. Anais eletrônicos... Istanbul:IEEE, 2016. p. 1-4. Disponível em: https://ieeexplore.ieee.org/document/7760744. Acesso em: 24 set. 2019.

BOAVENTURA, H. Profissões ligadas à tecnologia terão alto crescimento até 2023, aponta SENAI. Agência CNI de Notícias, 12 ago. 2019. Disponível em: 
https://noticias.portaldaindustria.com.br/noticias/educacao/profissoes-ligadas-a-tecnologiaterao-alto-crescimento-ate-2023-aponta-senai/. Acesso em: 22 nov. 2019.

BRASIL. Ministério da Ciência, Tecnologia, Inovações e Comunicações (MCTIC). Plano de CT\&I para Manufatura Avançada no Brasil (ProFuturo - Produção do Futuro). Brasília: MCTIC, 2017.

Disponível

em: https://www.mctic.gov.br/mctic/export/sites/institucional/tecnologia/tecnologias convergente s/arquivos/Cartilha-Plano-de-CTI WEB.pdf. Acesso em: 25 ago. 2019.

Ministério da Ciência, Tecnologia, Inovações e Comunicações (MCTIC). Plano de ação de ciência, tecnologia e inovação para tecnologias convergentes e habilitadoras. Brasília: $\quad$ MCTIC, 2020. Disponível em: https://www.mctic.gov.br/mctic/export/sites/institucional/tecnologia/Categoriadestaque/Arquivo/Plano-de-Acao-em-CTI-Manufatura-Avancada-VF.pdf. Acesso em: 27 abril 2020.

FIERGS. Rio Grande do Sul terá de qualificar mais de 800 mil trabalhadores em profissões industriais até 2023. FIERGS, 30 set. 2019. Disponível em: https://www.fiergs.org.br/noticia/rio-grande-do-sul-tera-de-qualificar-mais-de-800-miltrabalhadores-em-profissoes-industriais. Acesso em: 22 nov. 2019.

FORSCHUNGSUNION; ACATECH. Recommendations for implementing the strategic initiative INDUSTRIE 4.0. Final report of the Industrie 4.0 Working Group, 2013. Disponível em: https://en.acatech.de/publication/recommendations-for-implementing-thestrategic-initiative-industrie-4-0-final-report-of-the-industrie-4-0-working-group/. Acesso em: 25 out. 2019.

HEINDL, A. et al. Industrie 4.0: Possibilidades de colaboração com a cooperação para o desenvolvimento e a economia alemã na área de tecnologia/transferência de know-how para o Brasil. Bonn: 2016.2 GIZ, Disponível em: http://www.ahkbrasil.com/downloads/Arquivos/GIZ_Abschlussbericht_07_10_2016_FINAL \%20portugues FR clean.pdf. Acesso em: 27 ago. 2019.

INSTITUTO SENAI DE INOVAÇÃO EM SISTEMAS VIRTUAIS DE PRODUÇÃO. A visão das instituições: Indústria 4.0: Cenário, Desafios e Rede de Inovação. In: FIRJAN SENAI, FINEP (org.). Industria 4.0 no Brasil: oportunidades, perspectivas e desafios. Rio de Janeiro: [s.n.], 2019. p. 63. Disponível em: https://www.firjan.com.br/publicacoes/publicacoes-deinovacao/industria-4-0-no-brasil-oportunidades-perspectivas-e-desafios.htm\#pubAlign.

Acesso em: 30 out. 2019.

MDIC; ABDI. Agenda brasileira para a Indústria 4.0: O Brasil Preparado para os Desafios do Futuro, 2018. Disponível em: http://www.industria40.gov.br. Acesso em: 2 ago. 2019.

OLIVEIRA, J. D. Como podemos utilizar inteligência artificial no e-commerce? E-Commerce Brasil, 19 fev. 2020. Disponível em: https://www.ecommercebrasil.com.br/artigos/comopodemos-utilizar-inteligencia-artificial-no-e-commerce/. Acesso em: 03 maio 2020.

PEARSON, A. Criatividade e Inovação. 1ª edição, Brasil: Pearson, 2011. 
RAJNAI, Z.; KOCSIS, I. Labor market risks of industry 4.0, digitization, robots and AI. In: 15TH INTERNATIONAL SYMPOSIUM ON INTELLIGENT SYSTEMS AND INFORMATICS (SISY), 2017, Subotica. Anais eletrônicos... Subotica:IEEE, 2017. p. 343346. Disponível em: https://ieeexplore.ieee.org/document/8080580. Acesso em: 24 set. 2019.

RÜßMANN, M. et al. Industry 4.0: The Future of Productivity and Growth in Manufacturing Industries. Boston Consulting Group (BCG), 09 abril 2015. Disponível em: https://www.bcg.com/pt-

br/publications/2015/engineered_products_project business industry 4 future productivity growth manufacturing industries.aspx. Acesso em: 22 ago. 2019.

SILVA, V. L.; KOVALESKI, J. L.; PAGANI, R. N. Competências bases para o trabalho humano na Indústria 4.0. Revista FOCO, v. 12, n. 2, p. 112-129, jun. 2019. Disponível em: https://www.researchgate.net/publication/333658254_Competencias bases_para_o trabalho humano_na_Industria 40. Acesso em: 23 nov. 2019.

TESSARINI JUNIOR, G ; SALTORATO, P. Impactos da Indústria 4.0 na Organização do Trabalho: Uma Revisão Sistemática da Literatura. Revista Produção Online, Florianópolis, v. 18, n. 2, p. 743-769, jun. 2018. Disponível em: https://www.researchgate.net/publication/325803168 Impactos da industria 40 na organiza cao do trabalho uma revisao sistematica da_literatura. Acesso em: 22 nov. 2019.

YANG, G.-Z. et al. Combating COVID-19-The role of robotics in managing public health and infectious diseases. Science Robotics, Washington, v. 5, n. 40, 25 mar. 2020. Disponível em: https://robotics.sciencemag.org/content/5/40/eabb5589/tab-pdf. Acesso em: 07 mar. 2020.

\section{INDUSTRY 4.0 AND THE CHALLENGES OF EDUCATION}

Abstract: Industry 4.0, identified as the last industrial revolution, has been changing the industrial and social environment in several aspects, from production to the application of emerging technologies in people's daily lives. Advanced manufacturing, using intelligent machines that share information and make decisions without human intervention, determine the new skills and competencies needed by some professionals. In the Engineering area, innovations in industry 4.0 are present, as they involve technical and scientific knowledge, requiring a constant updating of industrial and market operations. In this context, this text presents research on challenges, skills and competences linked to the training of engineers to work in industry 4.0, emphasizing the need for educational renewal involving emerging technologies. In this direction, the work aims to provoke a reflection on the insertion of some concepts and technologies, used in industry 4.0, in the curriculum. As a result, it is identified how important it is to have the alignment of ideas and actions between government, companies, educational institutions and society so that a reformulation of teaching occurs through the continuous qualification of teachers and the structuring of school laboratories.

Keywords: Education, Industry 4.0, Engineering. 